\title{
RAS2 Regulates Growth and Pathogenesis in Fusarium graminearum
}

\author{
B. H. Bluhm, ${ }^{1}$ X. Zhao, ${ }^{2}$ J. E. Flaherty, ${ }^{3}$ J.-R. $X u,{ }^{2}$ and L. D. Dunkle ${ }^{1}$ \\ ${ }^{1}$ Crop Production \& Pest Control Research Unit, United States Department of Agriculture-Agricultural Research Service, \\ West Lafayette, IN 47907, U.S.A., ' Department of Botany and Plant Pathology, Purdue University, West Lafayette, IN 47907, \\ U.S.A.; and ${ }^{3}$ Department of Science and Mathematics, Coker College, 300 E. College Ave., Hartsville, SC 29550, U.S.A.
}

Submitted 21 December 2006. Accepted 11 January 2007.

Fusarium graminearum is a ubiquitous pathogen of cereal crops, including wheat, barley, and maize. Diseases caused by $\boldsymbol{F}$. graminearum are of particular concern because harvested grains frequently are contaminated with harmful mycotoxins such as deoxynivalenol (DON). In this study, we explored the role of Ras GTPases in pathogenesis. The genome of $F$. graminearum contains two putative Ras GTPase-encoding genes. The two genes ( $R A S 1$ and $R A S 2)$ showed different patterns of expression under different conditions of nutrient availability and in various mutant backgrounds. $R A S 2$ was dispensable for survival but, when disrupted, caused a variety of morphological defects, including slower growth on solid media, delayed spore germination, and significant reductions in virulence on wheat heads and maize silks. Intracellular cAMP levels were not affected by deletion of $R A S 2$ and exogenous treatment of the ras 2 mutant with cAMP did not affect phenotypic abnormalities, thus indicating that $R A S 2$ plays a minor or no role in CAMP signaling. However, phosphorylation of the mitogen-activated protein (MAP) kinase Gpmk1 and expression of a secreted lipase $(F G L 1)$ required for infection were reduced significantly in the ras 2 mutant. Based on these observations, we hypothesize that $R A S 2$ regulates growth and virulence in $F$. graminearum by regulating the Gpmk1 MAP kinase pathway.

Additional keywords: Gibberella zeae, head blight.

Fusarium graminearum (Schwabe), teleomorph Gibberella zeae (Schwein.) Petch, infects a broad range of crop plants, including wheat, barley, and maize (Bai and Shaner 2004; Goswami and Kistler 2004). During the 1990s, Fusarium head blight of wheat and barley reached epidemic proportions in North America, with losses estimated at $\$ 1.3$ billion in the United States (Bai and Shaner 2004). In addition to yield losses, infested grain often is contaminated with trichothecene mycotoxins, such as deoxynivalenol (DON), that are harmful

Corresponding author: Burton Bluhm; Telephone: +1.765 .496 .2416 ; Fax: +1.765.494.0363; E-mail: bluhm@ @urdue.edu

B. H. Bluhm and X. Zhao contributed equally to the work.

* The $\boldsymbol{e}$-Xtra logo stands for "electronic extra" and indicates that Figures 3 and 4 appear in color online.

This article is in the public domain and not copyrightable. It may be freely reprinted with customary crediting of the source. The American Phytopathological Society, 2007. to humans and animals (Desjardins et al. 1996; McMullen et al. 1997). DON is a potent inhibitor of protein synthesis in eukaryotic organisms (Kimura et al. 2001) and causes a variety of acute and chronic toxicoses when ingested by humans or animals (Etzel 2002; Joffe 1986). Due to the highly toxic nature of DON, regulatory agencies throughout the world have imposed strict guidelines and tolerances regarding its presence in grains and foods (van Egmond 2002).

Primary infection of wheat or maize by $F$. graminearum occurs during specific developmental stages of the hosts. Infection of wheat can occur from the beginning of anthesis until the dough stage of kernel development and initiates when ascospores or conidia are deposited on or inside flowering spikelets (Parry et al. 1995). After spore germination, the fungus penetrates through stomata or, more commonly, directly through floral bracts (Pritsch et al. 2000). From the infected floret, the fungus can spread up and down the spike and cause severe damage at conducive temperatures and humidity. In maize, infection is highest 1 to 6 days after silk emergence, at which time conidia deposited on silks germinate and the fungus grows down silk channels to colonize kernels (Sutton 1982). Unlike many plant-pathogenic fungi, $F$. graminearum does not produce specialized infection structures such as appressoria or haustoria during pathogenesis. Instead, colonization of tissues is facilitated primarily by the production of cell-wall-degrading enzymes such as cellulases, pectinases, and xylanases (Jenczmionka and Schafer 2005; Wanjiru et al. 2002).

The molecular mechanisms underlying pathogenesis in $F$. graminearum are complex and not fully understood. The production of trichothecene mycotoxins is not necessary for initial infection but is important for the spread of the fungus within colonized spikes (Bai et al. 2002; Desjardins et al. 2000; Harris et al. 1999; Proctor et al. 1995). Two mitogen-activated protein (MAP) kinase genes, $M G V 1$ and GPMK1, also are involved in pathogenesis in F. graminearum. Disruption of MGV1 substantially reduces DON production and virulence; $m g v l$ mutants rarely spread beyond the inoculated floret (Hou et al. 2002). Disruption of GPMK1 also impairs colonization of flowering wheat heads and the ability of the fungus to spread from inoculated florets to neighboring spikelets (Jenczmionka et al. 2003; Urban et al. 2003). Gpmk1 regulates the early induction of extracellular endoglucanase, xylanolytic, and proteolytic activities and is responsible for the overall induction of secreted lipolytic activities (Jenczmionka and Schafer 2005). Recently, FGL1, a gene encoding a secreted lipase, was identified as a virulence factor in F. graminearum (Voigt et al. 2005). Additionally, analyses of mutants generated by restriction enzyme-mediated (REMI) mutagenesis led to the discovery of four genes required for wild-type levels of virulence on wheat: CBL1 and $M S Y 1$, 
which are required for methionine synthesis; ZIF1, which encodes a putative b-ZIP transcription factor; and TBL1, a transducin $\beta$-subunit-like gene (Seong et al. 2005). Mutants of $F$. graminearum lacking homologs of the Cochliobolus heterostrophus genes CPS1, which belongs to a superfamily of genes encoding adenylate-forming enzymes, or NPS6, which is predicted to encode a nonribosomal peptide synthetase, show reduced virulence (Lee et al. 2005; Lu et al. 2003). However, the exact roles of these genes in pathogenesis have yet to be determined.

Ras GTPases are small, conserved GTP-binding proteins that cycle between active (GTP-bound) and inactive (GDPbound) conformations to act as switches in molecular circuits. In higher eukaryotes, Ras GTPases interact with a diverse assemblage of effector molecules to regulate divergent signaling pathways, thus serving as points of convergence in signal transduction cascades (Mitin et al. 2005). Although somewhat less is known about the functions of Ras proteins in fungi, Ras GTPases regulate processes such as hyphal growth, asexual and sexual reproduction, yeast-hyphal transitions, virulence, and programmed cell death (Lee and Kronstad 2002; Phillips et al. 2006; Toda et al. 1985; Waugh et al. 2002; Weeks and Spiegelman 2003). In some fungi, pathogenesis is regulated at least in part by Ras GTPases (Leberer et al. 2001; Lee and Kronstad 2002). Because of the diversity of signaling pathways regulated by Ras GTPases and their involvement with MAP kinase signal transduction networks in fungi (Ramezani-Rad 2003), Ras-encoding genes are excellent candidates for study as pathogenicity genes in $F$. graminearum.

The purpose of this study was to identify genes encoding Ras GTPases in F. graminearum and determine their involvement in pathogenesis. We found that the genome of F. gramin- earum contains two genes (RAS1 and RAS2) predicted to encode Ras GTPases. Disruption of RAS1 proved problematic, possibly due to it being essential for viability. RAS2 was dispensable for viability but disruption resulted in slower radial growth on solid media, delayed spore germination, and female sterility. The ras 2 mutant also was severely impaired in its ability to spread throughout wheat heads and corn silks despite growth and the production of DON in colonized kernels. Intracellular levels of cAMP were not affected in the ras 2 mutant and phenotypic abnormalities caused by disruption of $R A S 2$ were not ameliorated by exogenous cAMP treatment, thus indicating that $R A S 2$ plays a minor role in cAMP signaling. However, the ras 2 mutant was defective in activating the MAP kinase Gpmk1 and inducing the expression of FGL1, a secreted lipase involved in virulence. From these observations, we conclude that RAS2 interacts with the Gpmk1 MAP kinase signaling cascade required for plant infection and, thus, is a key regulatory component of virulence in $F$. graminearum.

\section{RESULTS}

\section{F. graminearum contains two genes encoding putative Ras GTPases.}

Ras GTPases are distinguished by two hallmark domains: a highly conserved amino terminal domain responsible for GTP binding and hydrolysis, and a hypervariable (HV) domain characterized by a cysteine-aliphatic-aliphatic-variable (CAAX) motif at the carboxy terminus that mediates membrane localization (Wright and Phillips 2006). A tBLASTx search with Ras-2 of Neurospora crassa as the query revealed the presence of two putative Ras GTPase-encoding genes in

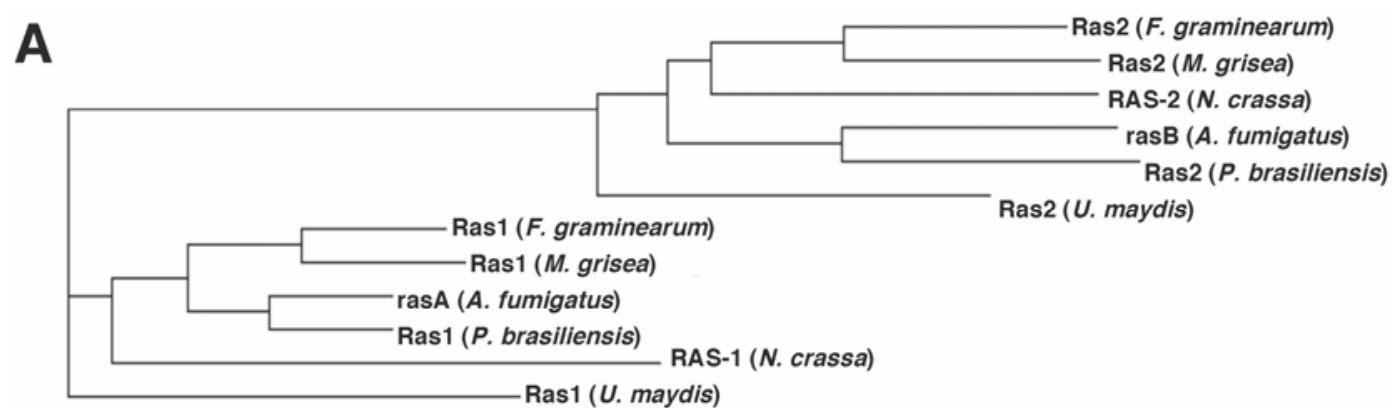

B

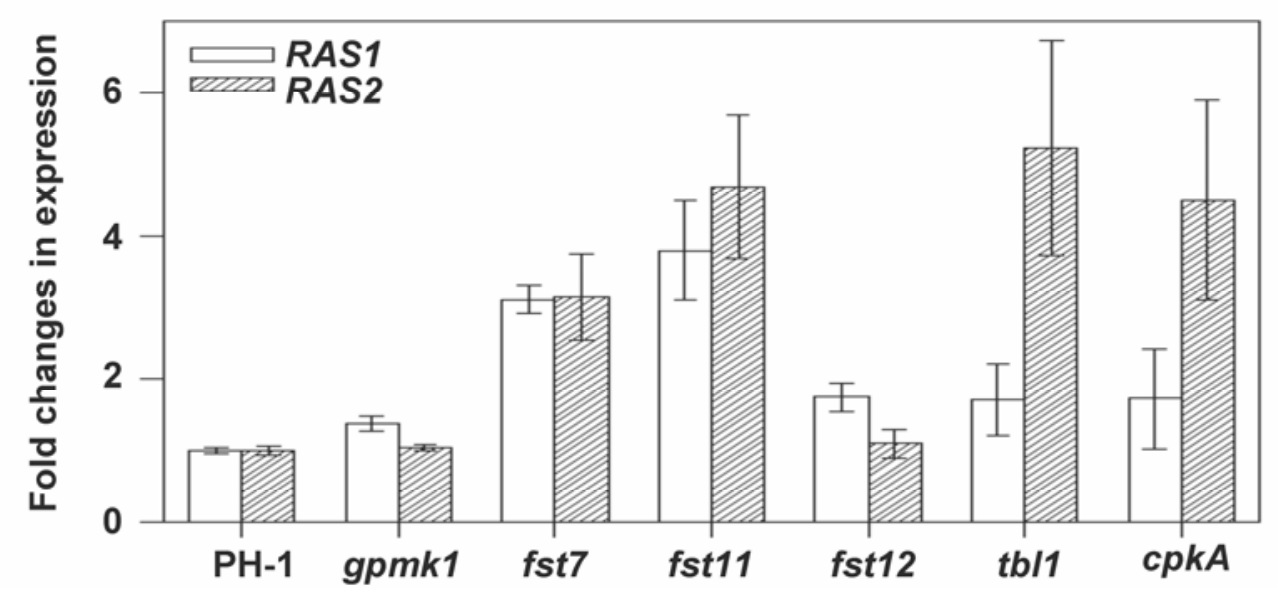

Fig. 1. Phylogenetic analysis of Ras proteins from Fusarium graminearum and other filamentous fungi. A, Amino acid sequences of Ras proteins were analyzed with the Clustal W program (Chenna et al. 2003) to create a phylogram tree. Homologs of Ras1 and Ras2 cluster separately in two distinct clades. B, Expression analyses of RAS1 and RAS2 in mutant backgrounds of $F$. graminearum as measured by real-time quantitative polymerase chain reaction. Expression levels are normalized to $\beta$-tubulin (TUB2) expression and are presented as fold changes relative to the wild type. 
the genome of $F$. graminearum, FG09778 (designated RAS1) and FG10114 (designated RAS2). RAS1 and RAS2 are predicted to encode proteins of 217 and 235 amino acids, respectively. The two predicted proteins share $51 \%$ identity, nearly all of which occurs over the GTP binding and hydrolysis domain. Both proteins display C-terminal CAAX motifs (CVLM for Ras1 and CVVL for Ras2) and significant divergence in the HV domains. A phylogenetic analysis of putative and previously characterized Ras proteins from $F$. graminearum and other filamentous fungi indicates the presence of two distinct phylogenetic clades (Fig. 1A). Ras2 from $F$. graminearum was $82 \%$ identical to a putative Ras GTPase from Magnaporthe grisea and $72 \%$ identical to Ras-2 from $N$. crassa. Among all fungal Ras proteins analyzed, the amino terminal domains were highly conserved; levels of amino acid conservation within HV domains accounted for the formation of the two clades (data not shown).

We examined the expression levels of RAS1 and RAS2 in a variety of environmental conditions and mutant backgrounds. From microarray data (Ulrich et al. 2006), we determined that carbon starvation did not significantly alter the expression of either RAS1 or RAS2, although nitrogen starvation resulted in a 2.5-fold increase in $R A S 2$ expression. Neither $R A S 1$ nor $R A S 2$

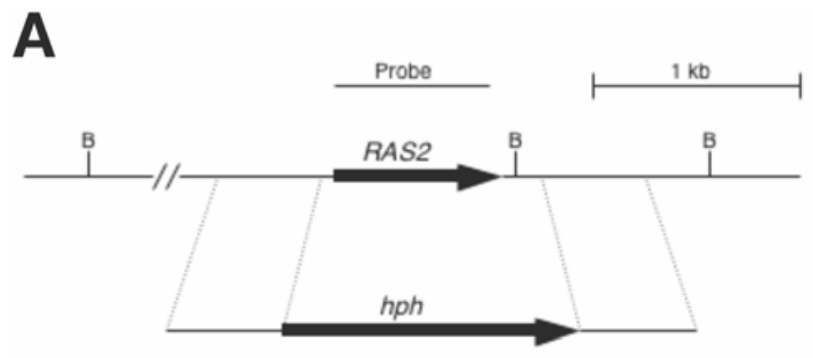

B

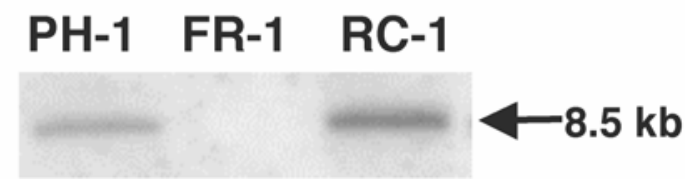

Fig. 2. RAS2 gene of Fusarium graminearum. A, The RAS2 gene was replaced with a hygromycin resistance cassette in strain $\mathrm{PH}-1$ via split-marker homologous recombination to create strain FR-1. For complementation, a $3.5-\mathrm{kb}$ region of the RAS2 locus was amplified by polymerase chain reaction, cloned into a vector containing a neomycin-resistance cassette, and transformed into strain FR-1 to create strain RC-1. B, Southern blot analysis was performed with a probe spanning the open reading frame of RAS2. A single band corresponding to $R A S 2$ was observed in the wild-type and complemented strains (RC-2 not shown) but was absent from the ras 2 mutant. expression is significantly affected by disruption of the MAP kinase Gpmk1 or Fst12, a transcription factor activated by the Gpmk1 MAP kinase signal transduction cascade (Fig. 1B). However, RAS1 and RAS2 show similar increases in expression when the MAPKK Fst7 or the MAPKKK Fst11 is disrupted (Fig. 1B), thus indicating that these genes directly or indirectly regulate $R A S$ gene expression. Interestingly, expression of $R A S 2$ was substantially higher than RAS1 in mutants of $T B L 1$, a transducin $\beta$-subunit-like gene, and of CpkA, which encodes a homolog of the protein kinase-encoding gene CPKA of $M$. grisea (Fig. 1B). These results indicate that the two Ras-encoding genes of $F$. graminearum are expressed differentially in various environmental conditions and genotypic backgrounds but may be coordinately regulated by components of the Gpmk1 MAP kinase pathway.

\section{Disruption of $R A S 2$.}

To characterize the functions of $R A S 2$, we utilized splitmarker replacement techniques (Fig. 2A). Repeated attempts to disrupt RAS1 failed; considering the high efficiency of gene disruption with this technique in $F$. graminearum (approximately $50 \%$ ), we conclude that RAS1 may be essential for viability. For $R A S 2$, four of six hygromycin-resistant transformants obtained tested positive for a double homologous gene-replacement event when screened by polymerase chain reaction (PCR) (data not shown). Southern analysis confirmed the deletion of the entire open reading frame of $R A S 2$ in isolate FR-1 (Fig. 2B). To confirm that phenotypic abnormalities of strain FR-1 were directly related to the deletion of the RAS 2 gene, we complemented the mutant by cloning the fulllength wild-type RAS2 allele and transforming it into the ras 2 mutant. The resulting neomycin-resistant transformants were screened by PCR to confirm the presence of the wildtype RAS2 gene (data not shown). Two neomycin-resistant isolates that tested positive by PCR were designated RC-1 and $\mathrm{RC}-2$, and the presence of RAS2 was confirmed by Southern blot analysis (Fig. 2B). Because of the high frequency of

Table 1. Growth and conidiation of the ras 2 mutant

\begin{tabular}{lccc}
\hline Strain & $\begin{array}{c}\text { Radial growth } \\
(\mathbf{m m})^{\mathbf{a}}\end{array}$ & $\begin{array}{c}\text { Dry weight } \\
(\mathbf{m g})^{\mathbf{b}}\end{array}$ & $\begin{array}{c}\text { Conidiation } \\
\left(\mathbf{1 0}^{\mathbf{4}} \mathbf{c o n i d i a} / \mathbf{m l}\right)^{\mathbf{b}}\end{array}$ \\
\hline PH-1 & $62 \pm 2.0$ & $120 \pm 6.3$ & $130 \pm 7.3$ \\
FR-1 & $18 \pm 1.1$ & $115 \pm 4.1$ & $137 \pm 8.9$ \\
RC-1 & $63 \pm 2.7$ & $122 \pm 8.0$ & $133 \pm 5.3$ \\
\hline
\end{tabular}

${ }^{\text {a }}$ Radial growth was measured as the diameter of colonies after 4 days incubation on potato-dextrose agar. Means and standard errors were calculated from three independent experiments.

b The dry weight of mycelia and number of conidia in $100 \mathrm{ml}$ of carboxymethyl-cellulose cultures were examined after incubating for 5 days. Standard error was calculated from three flasks per strain.
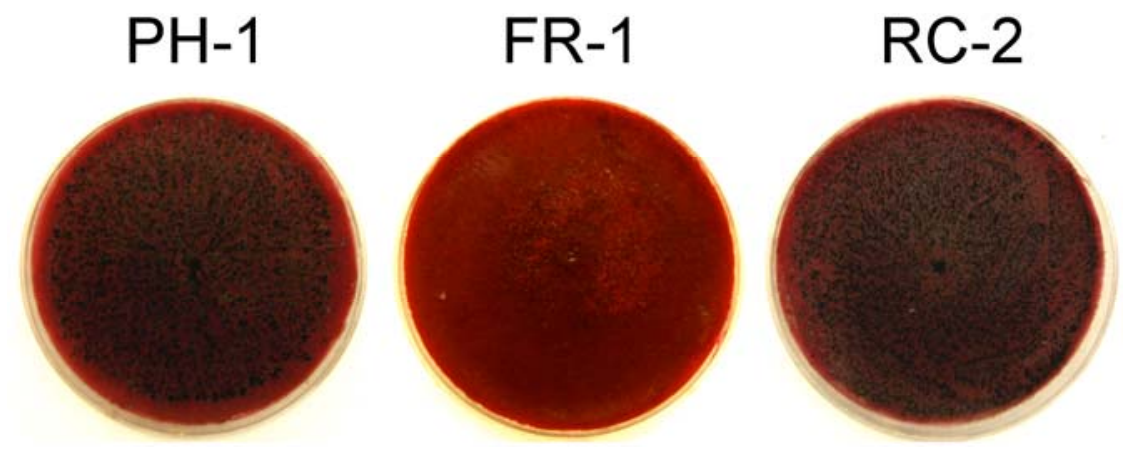

Fig. 3. Formation of perithecia in strains PH-1, FR-1, and RC-2 on carrot agar plates. Fungal strains were incubated on carrot agar for 4 weeks to induce formation of perithecia. Strain FR-1 was able to grow on the medium but failed to produce perithecia. 
homologous recombination in $F$. graminearum and the observation that the RAS2 gene was present on restriction fragments of similar size in the wild-type and complemented strains, we hypothesize that the complementation construct integrated at the RAS2 locus.

\section{$R A S 2$ is dispensable for conidiation but essential for sexual reproduction.}

In many fungi, Ras GTPases regulate aspects of conidiation or spore germination (Boyce et al. 2005; Fortwendel et al. 2004; Kana-uchi et al. 1997). In F. graminearum, disruption of $R A S 2$ had no significant effect on production of conidia (Table 1). However, the ras 2 strain failed to form perithecia on carrot agar plates (Fig. 3), indicating that the mutant is defective in female fertility. Strain RC-2 was normal in the production of perithecia and ascospores, thus confirming that the defect in female fertility was linked to the loss of the RAS2 gene.

\section{$R A S 2$ regulates hyphal growth.}

Disruption of Ras-encoding genes orthologous to RAS2 of $F$. graminearum results in morphological defects such as slower growth and colony abnormalities in several species of filamentous fungi (Boyce et al. 2005; Fortwendel et al. 2005; Kana-uchi et al. 1997). The rate of radial growth of the ras 2 mutant was significantly reduced when cultured on V8 agar plates (Fig. 4A and B). Similar reductions in growth were observed in the ras 2 mutant when cultured on potato-dextrose agar (PDA) plates (Table 1). In addition, the ras 2 mutant produced irregular colony margins and fewer aerial hyphae on solid media, including V8 (Fig. 4A), complete medium (CM), and PDA (data not shown). Growth of the complemented strain was indistinguishable from the wild-type $\mathrm{PH}-1$
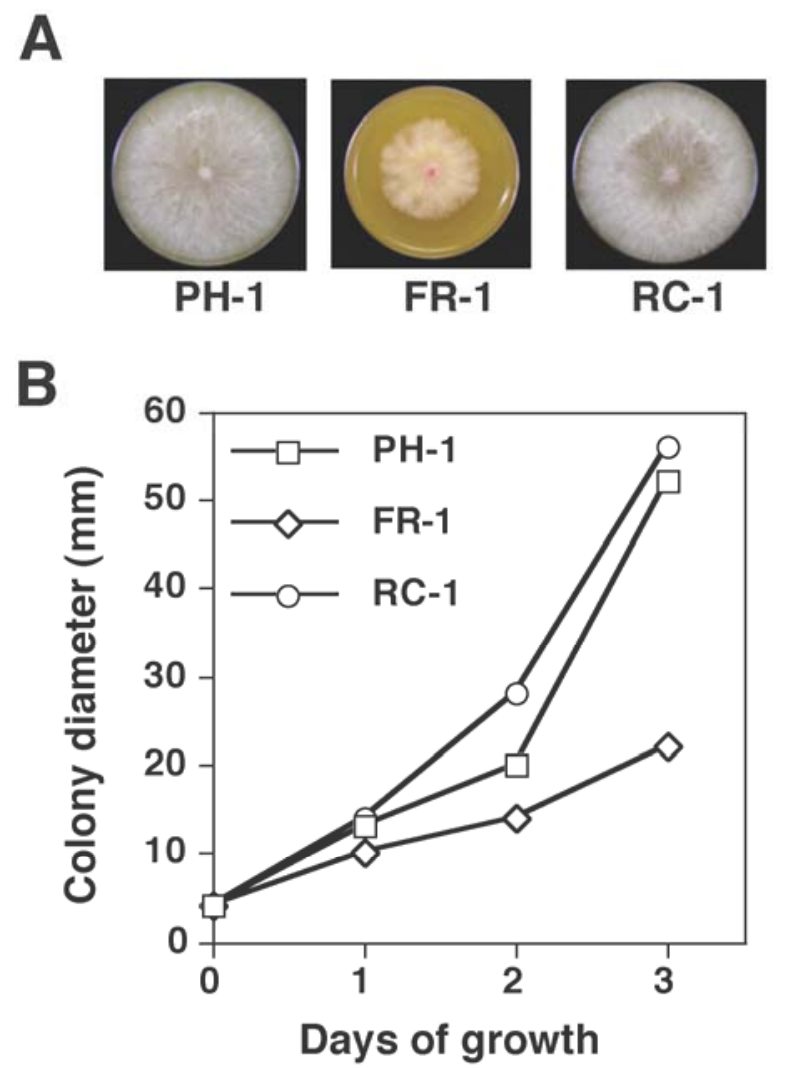

Fig. 4. RAS2 regulates growth in Fusarium graminearum. A, Colonies of the wild-type (PH-1), ras2 deletion mutant (FR-1), and complemented strain (RC-1) grown on V8 agar for 4 days. B, Growth rates on V8 agar. Measurements represent the average of three plates for each strain. on all media tested (Fig. 4A; data not shown), indicating that deletion of RAS2 accounted for the reduction in growth. Interestingly, similar amounts of fungal biomass accumulated when strains PH-1, FR-1, and RC-1 were grown in liquid carboxymethyl-cellulose (CMC) medium (Table 1), which indicates that the reduction in growth conferred by deletion of $R A S 2$ is conditional.

\section{$R A S 2$ is required for infection of wheat and maize.}

To determine the role of $R A S 2$ in pathogenesis, we inoculated wheat heads and maize silks with conidia from strains $\mathrm{PH}-1$, FR-1, and RC-1. On wheat heads inoculated with strains PH-1 and RC-1, scab symptoms developed first on inoculated spikelets and spread rapidly to uninoculated spikelets (Fig. 5A). In contrast, wheat heads inoculated with strain FR-1 developed scab symptoms only on inoculated spikelets. Disease index scores for the three strains showed a nearly 10-fold reduction in virulence for the FR-1 strain compared with strains PH-1 and RC-1 (Fig. 5B). Interestingly, despite a dramatic reduction in virulence, the FR-1 strain produced DON at an average of 180 ppm in scab kernels after 14 days of growth, which was similar to the average production of strains PH-1 (190 ppm) and RC-1 (300 ppm) after 14 days.

Infection of maize ears by $F$. graminearum occurs when the fungus colonizes living silk tissue and grows down silk channels. To determine whether RAS2 has a role in virulence on maize, we inoculated silk tissue with the PH-1, FR-1, and RC1 strains. Strains PH-1 and RC-1 were able to colonize silks, whereas the FR-1 strain was severely limited in its ability to grow on silk tissue (Fig. 5C). However, DON levels in inoculated maize kernels were similar among the three strains (data not shown).

To verify that the reduced virulence of the ras 2 mutant was not caused by an inability to grow on wheat or maize kernels, we quantified the growth of strains PH-1, FR-1, and RC-2 by measuring ergosterol levels in inoculated kernels. After 4 days of growth, ergosterol levels were similar among kernels inoculated with the three strains (Fig. 5D), and kernel colonization was visually indistinguishable among the three strains (data not shown). These results indicate that $R A S 2$ has an important role in pathogenesis on at least two of its host species independent of growth and DON biosynthesis.

\section{$R A S 2$ affects conidial germination but is not involved in cAMP signaling.}

In wild-type strains of $F$. graminearum, asexual spores (conidia) germinate within hours of exposure to favorable environmental conditions (Beyer et al. 2004). Germ tube emergence is typically bipolar, with a strong bias in favor of emergence from apical rather than internal cells of conidia (Harris 2005). Because conidia play key roles in diseases caused by $F$. graminearum, we determined the effect of the ras 2 mutation on spore germination. Disruption of RAS2 had no significant effect on patterns of germ tube emergence (data not shown) or the percentage of conidia that germinated (Fig. 6A). However, conidia of the ras 2 strain germinated more slowly than either the wild-type or the complemented strain (Fig. 6A), indicating that $R A S 2$ is involved in regulating germination.

In the $F$. graminearum genome, three proteins are predicted to contain Ras-association (RA) domains: homologs of yeast proteins Ste50, Ste11, and adenylate cyclase. In Saccharomyces cerevisiae and Candida albicans, adenylate cyclase is activated by Ras GTPases and disruption of Ras-encoding genes in either organism results in defective cAMP signaling (Fang and Wang 2006; Toda et al. 1985). To determine the role of $R A S 2$ in cAMP signaling in $F$. graminearum, we assayed the intracellular levels of cAMP in strains PH-1, FR-1, RC-1, and 
a mutant disrupted in the gene-encoding adenylate cyclase $(M A C 1)$ as a control. Intracellular cAMP levels were comparable among strains PH-1, FR-1, and RC-1 (Fig. 6B), which indicates that $R A S 2$ does not regulate cAMP production. To determine whether the delay in conidial germination of the ras 2 strain resulted from defects in cAMP signaling, germination experiments were repeated for the three strains in the presence of $10 \mathrm{mM}$ cAMP. Interestingly, exogenous cAMP did not suppress the slower germination of ras 2 conidia; in fact, treatment with cAMP delayed spore germination in all three strains (Fig. 6A). These results indicate that cAMP signaling is intact in the ras 2 strain and that $R A S 2$ plays only a minor, if any, role in cAMP-mediated processes.

$R A S 2$ is involved in the activation of Gpmk1 but not Mgv1.

Ras proteins are known to activate MAP kinase signal transduction pathways (Lee and Kronstad 2002; Masuda et al. 1995; Weeks and Spiegelman 2003). In F. graminearum, Mgv1 and Gpmk1 are two MAP kinases essential for pathogenesis. To determinate whether Ras2 is involved in the activation of Mgv1 or Gpmk1, we assayed the phosphorylation of these two MAP kinases in strains PH-1, FR-1, and RC-1. Total protein extracts isolated from vegetative hyphae of $\mathrm{PH}$ 1 showed low levels of Gpmk1 phosphorylation and even lower levels of Mgv1 activation (Fig. 7). In the FR-1 strain, phosphorylation of Gpmk1 was significantly reduced (Fig. 7), whereas the activation of Mgv1 was unaffected (Fig. 7). In strain RC-1, levels of Gpmk1 and Mgv1 phosphorylation were indistinguishable from strain PH-1 (Fig. 7). These data indicate that $R A S 2$ is required for wild-type levels of Gpmk1 phosphorylation.

\section{$R A S 2$ regulates the expression of $F G L 1$ during infection of wheat.}

In F. graminearum, Gpmk1 regulates the induction of secreted enzymes hypothesized to be required for pathogenesis (Jenczmionka and Schafer 2005). FGL1, which encodes a secreted lipase required for virulence, is induced during colonization of wheat (Voigt et al. 2005) through an unknown mechanism of induction. Based on our observation that RAS2 regulated the phosphorylation of Gpmk1, we hypothesized that expression of FGL1 was reduced in the ras 2 mutant during colonization of wheat. To test this hypothesis, we measured FGL1 expression in strains PH-1, FR-1, and RC-1 after 7 days of growth on wheat heads. In the ras 2 strain, expression of FGL1 was reduced by $50 \%$ compared with the wild-type or complemented strain (Fig. 8).

To determine whether FGL1 is regulated by Gpmk1, we measured expression of FGLl in the wild-type and gpmkl strains. Because mutants of Gpmkl are essentially apathogenic on wheat, we grew cultures of the wild-type and the gpmk1 strain in $2 \%$ wheat germ oil medium as described by Voigt and associates (2005). After $4 \mathrm{~h}$ of growth in the presence of wheat germ oil as the sole carbon source, expression of FGL1 was reduced by $50 \%$ in the gpmkl strain relative to the wild type (data not shown). We conclude that RAS2 modulates the activation of Gpmk1 to regulate the expression of FGL1 and possibly other genes encoding enzymes required for pathogenesis.

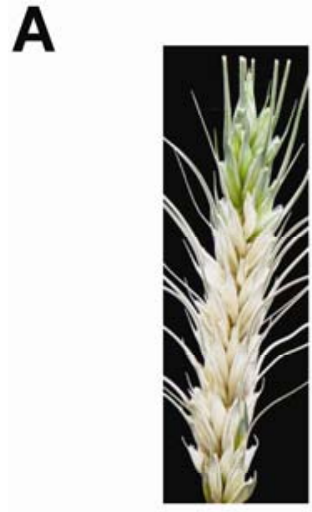

PH-1
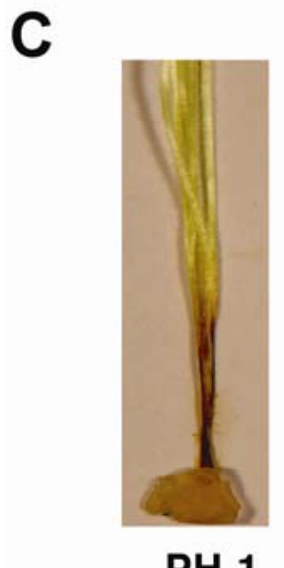

PH-1

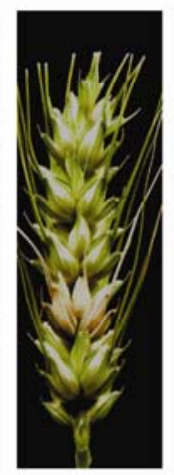

FR-1



FR-1

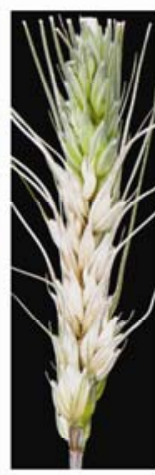

RC-1

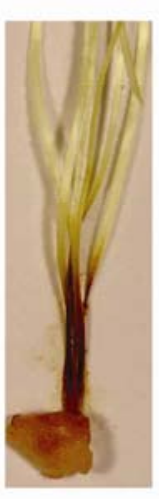

RC-1

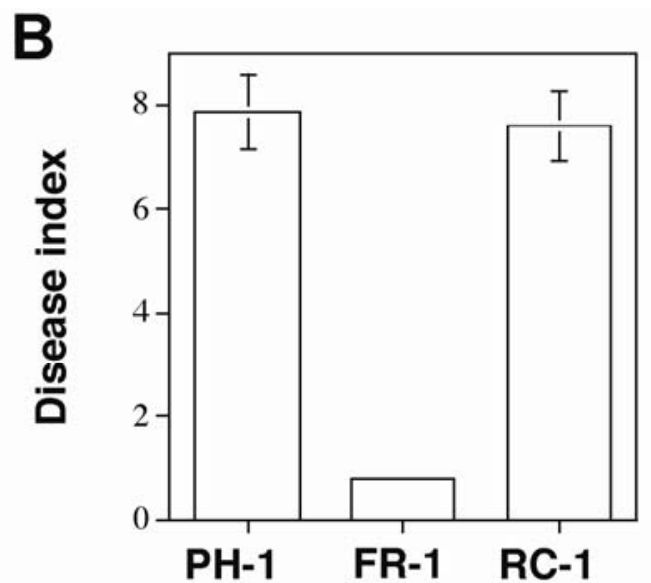

D

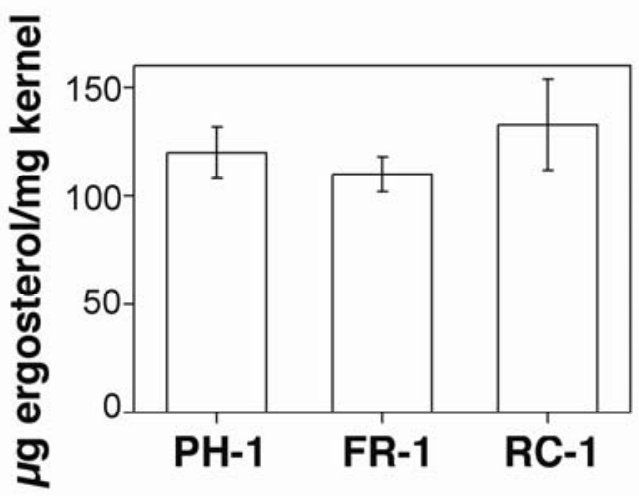

Fig. 5. The ras 2 mutant is defective in plant infection. A, Wheat head infection assays after 5 days of growth. B, Disease index scores were calculated from 19 to 20 plants for each fungal strain. Error bars represent the standard error of the mean. C, Corn silk infection assays after 5 days of growth. D, Growth of the three strains on individually inoculated wheat kernels after 7 days of growth as measured by ergosterol analysis. Error bars represent the standard error of the mean ergosterol concentration from five kernels. 


\section{DISCUSSION}

Ras proteins constitute a family of highly conserved regulatory proteins that are found in most, if not all, fungi. The genome of $F$. graminearum contains two genes encoding Ras GTPases, as do other filamentous ascomycetes for which sequenced genomes are available, including $M$. grisea, Aspergillus nidulans, and $N$. crassa. A phylogenetic analysis of Ras GTPases from these and other fungi indicates the presence of two distinct clades. However, it is difficult to predict the exact functions of Ras proteins based on phylogeny alone. Orthologs of $R A S 1$ have been implicated in regulating spore germination in several filamentous ascomycetes, including A. nidulans and Penicillium marneffei (Boyce et al. 2005; Fortwendel et al. 2005). In Colletotrichum trifolii, the causal agent of alfalfa anthracnose, disruption of the gene (Ct-ras) encoding an ortholog of RAS1 proved lethal, but expression of a dominantly active mutation of Ct-ras caused abnormal growth and differentiation ( $\mathrm{Ha}$ et al. 2003). The role of RASl in $F$. graminearum is unknown; however, the difficulties we encountered in knocking it out indicate that it may be an essential gene, as is its homolog in M. grisea (Park et al. 2006).

The functions of RAS2 orthologs are also diverse among filamentous fungi. In $A$. fumigatus, disruption of $r a s B$ causes a phenotype similar in many respects to that of the ras 2 mutant

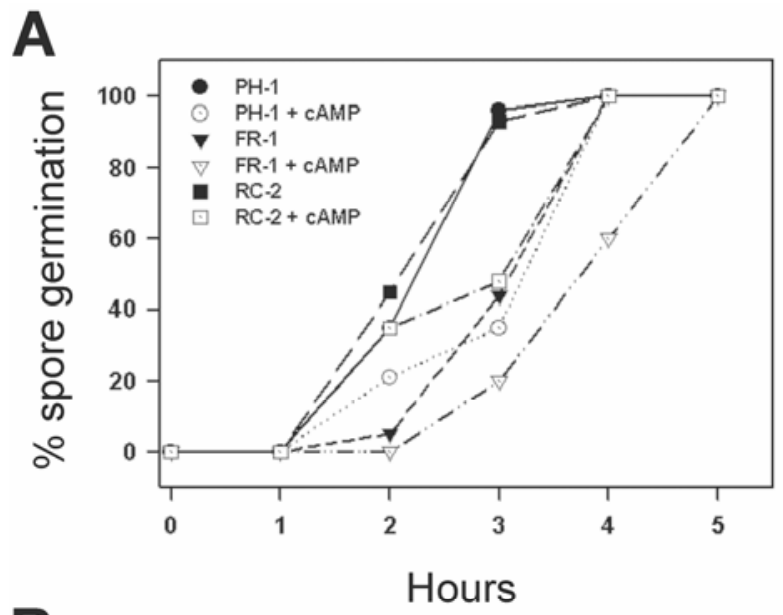

B

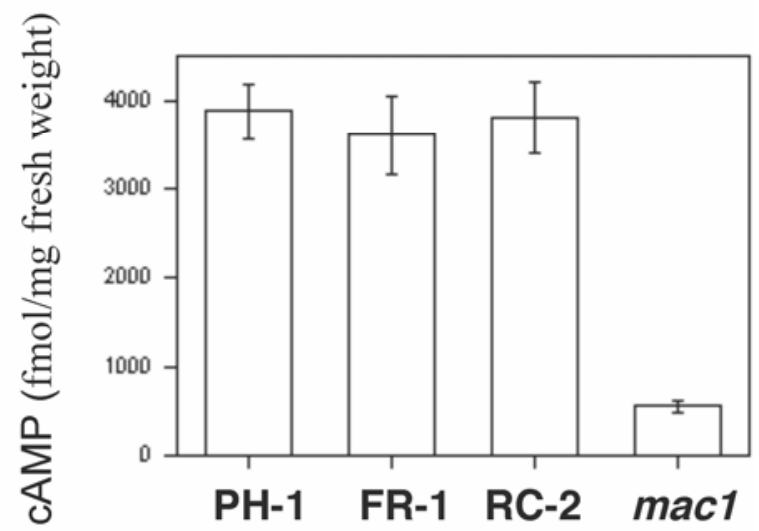

Fig. 6. $R A S 2$ does not regulate cAMP signaling. A, Germination experiments were performed with conidia from strains PH-1, FR-1, and RC-2 in the presence and absence of $10 \mathrm{mM}$ cAMP. Conidia of strain FR-1 germinated more slowly than $\mathrm{PH}-1$ or RC-2, and exogenous cAMP delayed germination in all three strains. B, Intracellular levels of cAMP were measured in tissue collected from 3-day-old liquid cultures of strains PH-1, FR-1, RC-2, and mac1. cAMP levels are presented in fmol/mg \pm standard error of three independent experiments. of $F$. graminearum; both mutants are reduced in growth and virulence, but are unaffected in conidiation (Fortwendel et al. 2005). However, disruption of the orthologous gene in $N$. crassa (Ras-2) results in a dramatic reduction in conidiation in addition to reduced growth (Kana-uchi et al. 1997). In $F$. graminearum, disruption of RAS2 had no effect on the production or viability of asexual spores; however, the inability of the ras 2 mutant to form perithecia indicates that $R A S 2$ regulates at least some aspects of sexual reproduction. However, the Spk1 MAP kinase pathway and mating processes are regulated by Ras1 in Schizosaccharomyces pombe (Papadaki et al. 2002). Ras GTPases also play important roles in regulating mating responses and sexual reproduction in several basidiomycetes, including Ustilago maydis, Cryptococcus neoformans, and Schizophyllum commune (Lee and Kronstad 2002; Schubert et al. 2006; Waugh et al. 2003). To our knowledge, RAS2 of $F$. graminearum is the first member of its clade demonstrated to play a role in plant infection or sexual reproduction in filamentous ascomycetes. If the functions of homologous genes are conserved in other plant-pathogenic ascomycetes, $R A S 2$ and its homologs may present novel targets in strategies to control fungal diseases.

To unravel the mechanism through which $R A S 2$ regulates pathogenesis in $F$. graminearum, we assayed the activation of the MAP kinases Mgv1 and Gpmk1. Although activation of Mgv1 was indistinguishable from the wild type in the ras 2 mutant, disruption of $R A S 2$ significantly impaired the activa-

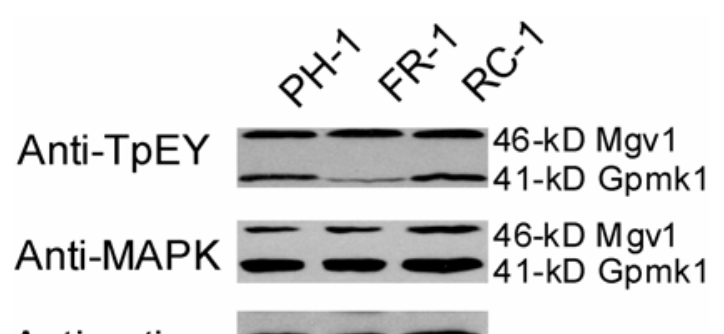

Anti-actin

Fig. 7. Activation of mitogen-activated protein kinases by Ras2. Western blot analysis of the expression and activation of Gpmk1 and Mgv1 in strains PH-1, FR-1, and RC-1. A 41-kDa (Gpmk1) band and a 46-kDa (Mgv1) band were observed in total protein isolated from all three strains (middle panel). When probed with an anti-TpEY antibody, bands corresponding to Mgv1 and Gpmk1 were observed in all three strains, but the Gpmk1 band was significantly reduced in the FR-1 strain (top panel). A similar amount of total protein was loaded in each lane, as determined by probing with an anti-actin antibody (bottom panel).

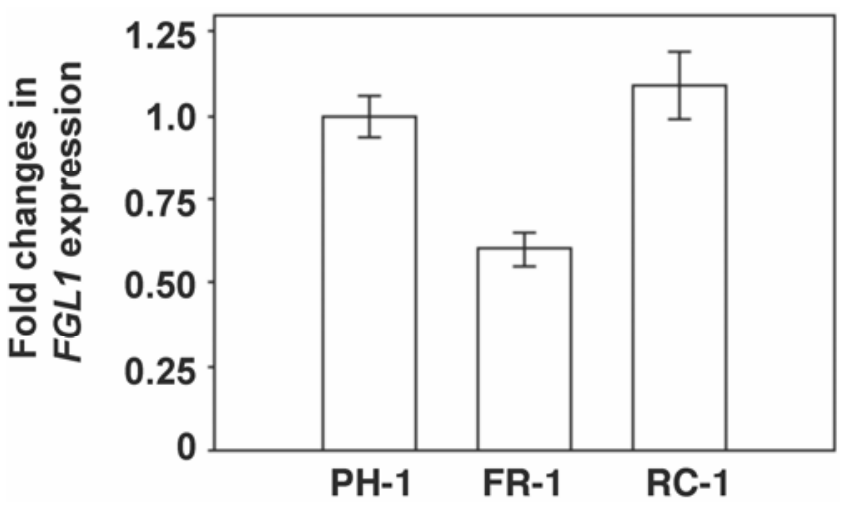

Fig. 8. RAS2 regulates the expression of $F G L 1$ during infection of wheat. Expression levels of the secreted lipase FGL1 were measured 7 days after inoculation of wheat heads by real-time quantitative polymerase chain reaction. Expression levels are normalized to $\beta$-tubulin (TUB2) expression and are presented as fold changes relative to the wild type. 
tion of Gpmk1. Phenotypically, the ras 2 mutant shares many similarities with strains disrupted in Gpmk1. Gpmk1 is essential for sexual reproduction and pathogenicity in $F$. graminearum (Jenczmionka et al. 2003; Urban et al. 2003), and its orthologs regulate plant infection processes in other fungal species (Mey et al. 2002; Ruiz-Roldan et al. 2001; Solomon et al. 2005; Xu 2000; Xu and Hamer 1996). During infection of wheat, both the ras 2 and gpmk1 mutants are significantly reduced in virulence despite producing DON in inoculated wheat kernels. Gpmk1 was demonstrated to regulate the secretion of lipolytic activities that are induced in the presence of wheat germ oil and are hypothesized to be involved in virulence (Jenczmionka and Schafer 2005). Recently, a gene encoding a secreted lipase $(F G L 1)$ was identified as a virulence factor in $F$. graminearum; in a manner similar to ras 2 and gpmkl mutants, the fgll mutant is unable to spread from colonized wheat kernels and fails to infect maize ears (Voigt et al. 2005). We found that expression of FGL1 is reduced by $50 \%$ in the ras 2 mutant compared with the wild type during growth on wheat heads, and expression of FGLl also is substantially lower in the gpmkl mutant relative to the wild type in the presence of wheat germ oil. We hypothesize that $R A S 2$ regulates the induction of extracellular enzymes required for virulence, and that this regulation occurs through activation of Gpmk1. In Saccharomyces cerevisiae, the MAP KKK Ste11 is regulated by Ste50, which contains an RA domain required for delivery to the plasma membrane (Truckses et al. 2006). In M. grisea, Mst50 and Mst11 (homologs of yeast Ste50 and Ste11, respectively) are important for activating the Pmkl MAP kinase pathway (Zhao et al. 2005) and interact with Ras1 and Ras2 in yeast two-hybrid assays (Park et al. 2006). Of the three proteins containing RA domains predicted to be present in $F$. graminearum, two are putative orthologs of Mst11 and Mst50. We recently found that Mst11 is essential for activating Pmk1 and plant infection in M. grisea (Park et al. 2006). Conceivably, Ras2 may interact with homologs of Ste50 or Ste11 to regulate the induction of Gpmk1 in F. graminearum.

The precise mechanism through which structurally similar Ras proteins regulate distinct signaling pathways in fungi is unclear. The proteins predicted to be encoded by RAS1 and RAS2 of $F$. graminearum differ primarily in their $\mathrm{HV}$ domains, which facilitate insertion into the inner leaflet of the plasma membrane. In mammalian systems, accumulating evidence indicates that Ras proteins are specifically targeted to discrete microdomains of the plasma membrane (i.e., lipid rafts) (Parton and Hancock 2004). In the emerging model, lipid rafts act as sorting mechanisms that facilitate the recruitment or co-localization of Ras GTPases and other proteins involved in signal transduction. Among fungi, lipid rafts were first demonstrated to occur in $S$. cerevisiae (Bagnat et al. 2000) and recently have been implicated in the concentration of virulence determinants in $C$. neoformans (Siafakas et al. 2006), although the interaction of fungal Ras proteins with lipid rafts has not been demonstrated. Another mechanism that could explain the specificity of Ras signaling is interactions with effector proteins such as guanine nucleotide exchange factors (GEFs) and GTPase-activating proteins (GAPs). Ras GEFs promote the formation of active Ras-GTP by stimulating GDP/GTP exchange, whereas Ras GAPs promote the formation of inactive Ras-GDP by increasing the rate of GTP hydrolysis (Mitin et al. 2005). Ras GEFs and Ras GAPs have been characterized in $S$. cerevisiae and other fungi (Camus et al. 1994; Müller et al. 2003; Schubert et al. 2006; Tanaka et al. 1990). The genome of $F$. graminearum is predicted to contain at least four genes encoding proteins with Ras GEF domains and two genes encoding proteins containing Ras GAP domains. Functional analysis of each of these genes will be required to determine their role, if any, in pathogenesis.
Based on our findings, we hypothesize that Ras2 of $F$. graminearum activates the Gpmk1 pathway to modulate signal transduction networks underlying pathogenesis in F. graminearum, including the induction of cell-wall-degrading enzymes such as Fgl1. The characterization of RAS2 provides a new avenue of study to unravel the complex interactions that control pathogenesis in $F$. graminearum. Perhaps most significantly, Ras 2 and regulators of Ras 2 activity such as lipid microdomains and Ras effector molecules could provide novel targets to control diseases caused by $F$. graminearum and possibly other plant-pathogenic fungi.

\section{MATERIALS AND METHODS}

\section{Fungal strains and growth conditions.}

F. graminearum wild-type strain PH-1 (NRRL 31084) and transformants generated in this study (including FR-1, RC-1, and $\mathrm{RC}-2$ described below) were maintained at $25^{\circ} \mathrm{C}$ on $\mathrm{V} 8$ agar or cultured in liquid CMC medium for conidiation (Hou et al. 2002). Transformants were selected on CM supplemented with hygromycin B (Calbiochem, La Jolla, CA, U.S.A.) at 150 $\mu \mathrm{g} / \mathrm{ml}$ or neomycin (Amresco, Solon, OH, U.S.A.) at 200 $\mu \mathrm{g} / \mathrm{ml}$. Growth and morphology were evaluated by culturing strains on V8, CMC, or PDA (BD, Sparks, MD, U.S.A.). Diameters of colonies formed on 150-by-15-mm petri plates were measured daily for 3 days (Hou et al. 2002). Formation of perithecia was assayed on modified carrot agar plates as described by Bowden and Leslie (1999). Intracellular cAMP was extracted from mycelia collected from 3-day-old 5× YEG (yeast extract glucose) liquid cultures as previously described (Nishimura et al. 2003) and quantified with the cAMP enzyme immunoassay system (Amersham Pharmacia Biotech, Piscataway, NJ, U.S.A.) following the manufacturer's instructions.

\section{Generation of a $R A S 2$ gene replacement mutant.}

A split-marker gene-disruption approach (Catlett et al. 2003) was used to delete RAS2 (FG10114). A 556-bp fragment upstream from RAS2 was amplified with primers EcoRAS (5' CT TGGAATTCGACCTGGACTTG 3') and FseRAS (5' CATTC GGCCGGCCATG $3^{\prime}$ ) to incorporate EcoRI and FseI restriction sites (underlined) into the product. A 560-bp fragment downstream from $R A S 2$ was amplified with primers AscRAS (5' CAAATCGGCGCGCCAACAACGA 3') and BglRAS (5' CGACAATAGATCTTCATGTCTGGCG $3^{\prime}$ ) to incorporate $A s c I$ and $B g l I I$ sites (underlined). These products were sequentially cloned into pCX63 (Zhao et al. 2004) to generate the $R A S 2$ gene replacement construct pHYG-RAS. Products for split-marker disruption were generated by PCR from pHYGRAS with primer sets EcoRAS and fHYG (5' AGGGTGTCAC GTTGCAAG $3^{\prime}$ ) as well as BglRAS and rHYG (5' ATGCCTC CGCTCGAAGT $3^{\prime}$ ). In all, $5 \mu \mathrm{g}$ of each split-marker product were purified and used for transformation. Hygromycin-resistant colonies were screened by PCR with primers RasS1 ( $5^{\prime} \mathrm{C}$ CGTCCGGTAACAGCTCTG 3') and Hyg5R (5' CGATCAGA AACTTCTCGACAG $3^{\prime}$ ) and with primers RasS2 (5' TCGAG CGTAGCGTTCCGATG 3') and Hyg3F (5'CGATAGTGGAA ACCGACG $3^{\prime}$ ). An isolate that tested positive with both primer sets (designated FR-1) was confirmed by Southern analysis as described below.

For complementation, a $3.5-\mathrm{kb}$ segment of the RAS2 gene encompassing the open reading frame, $1.7 \mathrm{~kb}$ of the promoter region, and $0.9 \mathrm{~kb}$ of the terminator was amplified with primers RasCF (5' AATAGCGGCCGCGTTCAGTGTGGCAGAG A $3^{\prime}$ ) and RasCR (5' ATAAGCGGCCGCAGTCGCTATCTTA GTGGA $3^{\prime}$ ) to incorporate NotI restriction sites (underlined) at both ends of the product. Primers NeoF (5' AATACCCGGG ATTAACGCTTACAATTTCCATTCGCCA $\left.3^{\prime}\right)$ and NeoR (5' A 
TAACCCGGGAATAGGAACTTCGGAATAGGAACTTCA 3') were used to amplify the neomycin resistance cassette from pSM334 (Hou et al. 2002) and incorporate XmnI restriction sites (underlined). The resulting $2.1-\mathrm{kb}$ fragment was cloned into an XmnI site on the pBC KS backbone to create pHZ100, which then was digested with NotI for the insertion of the Ras complementation fragment to create pRasCOMP. Strain FR-1 was transformed with pRasComp to create strains RC-1 and RC-2.

Southern analysis was performed according to standard protocols as described by Sambrook and Russell (2001). Genomic DNA $(10 \mu \mathrm{g})$ from strains PH-1, FR-1, and RC-1 was digested with BamHI, separated on a $0.7 \%$ agarose gel, and transferred to nylon membranes. High-stringency hybridization was performed with a ${ }^{32} \mathrm{P}$-labeled probe spanning the open reading frame of RAS2. The probe was amplified from genomic DNA from strain PH-1 using primers RasPrbF (5' ATGGCCGGCCG AATGGTACTAT $3^{\prime}$ ) and RasPrbR (5' CATAATACCACGCAT TTAGACT 3').

\section{Analyses of gene expression.}

The expression of RASl (forward primer: 5' GTGTCACGA CAGGAGGGAGAG 3', reverse primer: 5' GCCTTGTCGAC ATTGATTCGAGACT 3'), RAS2 (forward primer: 5' TTTGTT GAAACTTACGACCCCACGAT 3', reverse primer: 5' GCGG TGTCCAGTACTTCGAGC $3^{\prime}$ ), and $F G L 1$ (forward primer: 5' GCATCCTTCACGGATCCAAGACTG 3', reverse primer: 5' AGTTGCGGATGTTGATGCTACCA 3') was measured by realtime quantitative PCR (qPCR). RNA was extracted from wheat heads or liquid cultures with Trizol reagent (Invitrogen, Carlsbad, CA, U.S.A.) and cDNA template for qPCR was prepared as described previously (Bluhm and Woloshuk 2005). All expression data were normalized to TUB2 expression (forward primer: 5' GTCAGTGCGGTAACCAAATCGGT 3', reverse primer: 5' CTCAGAGGTGCCGTTGTAAACACC $3^{\prime}$ ) and relative changes in gene expression were calculated with the comparative $\mathrm{Ct}$ method (Applied Biosystems, Foster City, CA, U.S.A.).

\section{Corn silk and wheat infection assays.}

Fresh corn silks were inoculated with mycelia collected from 5-day-old V8 agar cultures and incubated at $25^{\circ} \mathrm{C}$ in a moisture chamber for 4 to 5 days as described by Seong and associates (2005). Five- to six-week-old plants of wheat cv. Norm were used in infection assays with conidia collected from 5- to 7-day-old CMC cultures as described by Hou and associates (2002). The third full-sized spikelet from the base of the inflorescence was injected with $10 \mu \mathrm{l}$ of the conidium suspension $\left(10^{6} \mathrm{conidia} / \mathrm{ml}\right)$. After inoculation, wheat heads were covered with a small plastic bag for 2 days to maintain humidity. Symptomatic spikelets in each head were counted 14 days after inoculation and disease index scores were calculated as described by Hou and associates (2002). The number of inoculated wheat heads per treatment was 9 or 10 for each test and all tests were repeated at least four times.

\section{Analysis of ergosterol and DON production.}

Ergosterol levels were measured in individually inoculated wheat kernels after 7 days of growth as described by Bluhm and Woloshuk (2005). For DON analysis, wheat spikelets (five per fungal strain) were inoculated as described above and kernels were analyzed individually. Maize kernels were prepared and inoculated essentially as described by Bluhm and Woloshuk (2005). Autoclaved kernels ( $5 \mathrm{~g}$ ) were placed in sterile scintillation vials, inoculated with $10^{4}$ conidia, and incubated at $24^{\circ} \mathrm{C}$ for 10 days. Maize and wheat kernels were extracted and analyzed as described by Lauren and Greenhalgh (1987) with minor modifications. Kernels were ground under liquid $\mathrm{N}_{2}$ and extracted with acetonitrile/water (85:15, vol/vol) with shaking for $2 \mathrm{~h}$. Liquid chromatography was performed with a Beckman high-performance liquid chromatograph, mobile phase acetonitrile/water $(10: 90, \mathrm{vol} / \mathrm{vol})$ at $1 \mathrm{ml} / \mathrm{min}$, with detector wavelength set at $218 \mathrm{~nm}$. The retention time for DON was 8.1 min. Quantification was performed by comparing peak areas of samples to those of an external standard of DON (Sigma, St. Louis).

\section{Western blot analysis.}

Total proteins were isolated from vegetative hyphae as described previously (Bruno et al. 2004) and separated on an $8 \%$ sodium dodecyl sulfate polyacrylamide gel electrophoresis gel (approximately $20 \mu \mathrm{g} / \mathrm{lane}$ ) and transferred to nitrocellulose membranes. Antigen-antibody detection was performed with the ECL Supersignal System (Pierce, Rockford, IL, U.S.A.). The Mgv1 and Gpmk1 MAP kinases and their levels of TEY phosphorylation were detected with the PhophoPlus p44/42 MAP kinase antibody kit (Cell Signaling Technology, Beverly, MA, U.S.A.) following the manufacturer's instructions.

\section{GenBank accession numbers.}

Accession numbers were AAO19640 for Ras1 and AAO19639 for Ras2 of U. maydis, AAQ94235 for RasA and AAP94030 for RasB of A. fumigatus, CAA37612 for Ras-1 and BAA03708 for Ras-2 of N. crassa, AAQ04628 for Ras1 and AAT27446 for Ras2 of Paracoccidioides brasiliensis, XP_369310 for Ras1 and XP_364654 for Ras2 of M. grisea, and AY805204 for Exserohilum turcicum EST 1A11.

\section{ACKNOWLEDGMENTS}

Product names are necessary to report factually on available data; however, the United Stated Department of Agriculture (USDA) neither guarantees nor warrants the standard of the product, and the use of the names implies no approval of the product to the exclusion of others that may also be suitable. This work was partly supported by a grant from the United States Wheat and Barley Scab initiative and a grant from the USDA National Research Initiative (\#2003-01130) to J. Xu. Published as ARP no. 200618031 of the Purdue University Agricultural Experiment Station. We thank S.-L. Ding for assistance with microarray analyses, Y.-H. Dong for DON measurements, and Z. Hou for assisting with complementation analyses.

\section{LITERATURE CITED}

Bagnat, M., Keranen, S., Shevchenko, A., and Simons, K. 2000. Lipid rafts function in biosynthetic delivery of proteins to the cell surface in yeast. Proc. Natl. Acad. Sci. U.S.A. 97:3254-3259

Bai, G. H., and Shaner, G. 2004. Management and resistance in wheat and barley to Fusarium head blight. Annu. Rev. Phytopathol. 42:135-161.

Bai, G. H., Desjardins, A. E., and Plattner, R. D. 2002. Deoxynivalenolnonproducing Fusarium graminearum causes initial infection, but does not cause disease spread in wheat spikes. Mycopathologia 153:91-98.

Beyer, M., Roding, S., Ludewig, A., and Verreet, J.-A. 2004. Germination and survival of Fusarium graminearum macroconidia as affected by environmental factors. J. Phytopathol. 152:92-97.

Bluhm, B. H., and Woloshuk, C. P. 2005. Amylopectin induces fumonisin B1 production by Fusarium verticillioides during colonization of maize kernels. Mol. Plant-Microbe Interact. 18:1333-1339.

Bowden, R. L., and Leslie, J. F. 1999. Sexual recombination in Gibberella zeae. Phytopathology 89:182-188.

Boyce, K. J., Hynes, M. J., and Andrianopoulos, A. 2005. The Ras and Rho GTPases genetically interact to co-ordinately regulate cell polarity during development in Penicillium marneffei. Mol. Microbiol. 55:1487-1501.

Bruno, K. S., Tenjo, F., Li, L., Hamer, J. E., and Xu, J. R. 2004. Cellular localization and role of kinase activity of PMK1 in Magnaporthe grisea. Eukaryot. Cell 3:1525-1532.

Camus, C., Boy-Marcotte, E., and Jacquet, M. 1994. Two subclasses of guanine exchange factor (GEF) domains revealed by comparison of activities of chimeric genes constructed from CDC25, SDC25 and BUD5 in Saccharomyces cerevisiae. Mol. Gen. Genet. 245:167-176.

Catlett, N. L., Lee, B., Yoder, O. C., and Turgeon, B. G. 2003. Split-marker 
recombination for efficient targeted deletion of fungal genes. Fungal Genet. Newsl. 50:9-11.

Chenna, R., Sugawara, H., Koike, T., Lopez, R., Gibson, T. J., Higgins, D. G., and Thompson, J. D. 2003. Multiple sequence alignment with the Clustal series of programs. Nucleic Acids Res. 31:3497-3500.

Desjardins, A. E., Proctor, R. H., Bai, G. H., McCormick, S. P., Shaner, G., Buechley, G., and Hohn, T. M. 1996. Reduced virulence of trichothecene-nonproducing mutants of Gibberella zeae in wheat field tests. Mol. Plant-Microbe Interact. 9:775-781.

Desjardins, A. E., Bai, G. H., Plattner, R. D., and Proctor, R. H. 2000 Analysis of aberrant virulence of Gibberella zeae following transformation-mediated complementation of a trichothecene-deficient (Tri5) mutant. Microbiology 146:2059-2068.

Etzel, A. 2002. Mycotoxins. JAMA 287:425-427.

Fang, H. M., and Wang, Y. 2006. RA domain-mediated interaction of Cdc35 with Ras1 is essential for increasing cellular cAMP level for Candida albicans hyphal development. Mol. Microbiol. 61:484-496.

Fortwendel, J. R., Panepinto, J. C., Seitz, A. E., Askew, D. S., and Rhodes, J. C. 2004. Aspergillus fumigatus rasA and rasB regulate the timing and morphology of asexual development. Fungal Genet. Biol. 41:129-139.

Fortwendel, J. R., Zhao, W., Bhabhra, R., Park, S., Perlin, D. S., Askew, D. S., and Rhodes, J. C. 2005. A fungus-specific Ras homolog contributes to the hyphal growth and virulence of Aspergillus fumigatus. Eukaryot. Cell 4:1982-1989.

Goswami, R. S., and Kistler, H. C. 2004. Heading for disaster: Fusarium graminearum on cereal crops. Mol. Plant Pathol. 5:515-525.

Ha, Y. S., Memmott, S. D., and Dickman, M. B. 2003. Functional analysis of Ras in Colletotrichum trifolii. FEMS (Fed. Eur. Microbiol. Soc.) Microbiol. Lett. 226:315-321.

Harris, S. D. 2005. Morphogenesis in germinating Fusarium graminearum macroconidia. Mycologia 97:880-887.

Harris, L. J., Desjardins, A. E., Plattner, R. D., Nicholson, P., Butler, G., Young, J. C., Weston, G., Proctor, R. H., and Hohn, T. M. 1999. Possible role of trichothecene mycotoxins in virulence of Fusarium graminearum on maize. Plant Dis. 83:954-960.

Hou, Z. M., Xue, C. Y., Peng, Y. L., Katan, T., Kistler, H. C., and Xu, J. R. 2002. A mitogen-activated protein kinase gene (MGV1) in Fusarium graminearum is required for female fertility, heterokaryon formation, and plant infection. Mol. Plant-Microbe Interact. 15:1119-1127.

Jenczmionka, N. J., and Schafer, W. 2005. The Gpmk1 MAP kinase of Fusarium graminearum regulates the induction of specific secreted enzymes. Curr. Genet. 47:29-36.

Jenczmionka, N. J., Maier, F. J., Losch, A. P., and Schafer, W. 2003. Mating, conidiation and pathogenicity of Fusarium graminearum, the main causal agent of the head-blight disease of wheat, are regulated by the MAP kinase GPMK1. Curr. Genet. 43:87-95.

Joffe, A. Z. 1986. Fusarium Species: Their Biology and Toxicology. Wiley and Sons, New York.

Kana-uchi, A., Yamashiro, C. T., Tanabe, S., and Murayama, T. 1997. A ras homologue of Neurospora crassa regulates morphology. Mol. Gen. Genet. 254:427-432.

Kimura, M., Anzai, H., and Yamaguchi, I. 2001. Microbial toxins in plantpathogen interactions: Biosynthesis, resistance mechanisms, and significance. J. Gen. Appl. Microbiol. 47:149-160

Lauren, D., R., and Greenhalgh, R. 1987. Simultaneous analysis of nivalenol and deoxynivalenol in cereals by liquid chromatography. J. Assoc. Off. Anal. Chem. 70:479-483.

Leberer, E., Harcus, D., Dignard, D., Johnson, L., Ushinsky, S., Thomas, D. Y., and Schroppel, K. 2001. Ras links cellular morphogenesis to virulence by regulation of the MAP kinase and cAMP signaling pathways in the pathogenic fungus Candida albicans. Mol. Microbiol. 42:673-687.

Lee, B. N., Kroken, S., Chou, D. Y. T., Robbertse, B., Yoder, O. C., and Turgeon, B. G. 2005. Functional analysis of all nonribosomal peptide synthetases in Cochliobolus heterostrophus reveals a factor, NPS6, involved in virulence and resistance to oxidative stress. Eukaryot. Cell 4:545-555.

Lee, N., and Kronstad, J. W. 2002. ras2 Controls morphogenesis, pheromone response, and pathogenicity in the fungal pathogen Ustilago maydis. Eukaryot. Cell 1:954-966.

Lu, S. W., Kroken, S., Lee, B. N., Robbertse, B., Churchill, A. C. L., Yoder, O. C., and Turgeon, B. G. 2003. A novel class of gene controlling virulence in plant pathogenic ascomycete fungi. Proc. Natl. Acad. Sci. U.S.A. 100:5980-5985.

Masuda, T., Kariya, K., Shinkai, M., Okada, T., and Kataoka, T. 1995. Protein kinase Byr2 is a target of Ras1 in the fission yeast Schizosaccharomyces pombe. J. Biol. Chem. 270:1979-1982.

McMullen, M., Jones, R., and Gallenberg, D. 1997. Scab of wheat and barley: A re-emerging disease of devastating impact. Plant Dis. 81:13401348

Mey, G., Oeser, B., Lebrun, M. H., and Tudzynski, P. 2002. The biotrophic, non-appressorium-forming grass pathogen Claviceps purpurea needs a FUS3/PMK1 homologous mitogen-activated protein kinase for colonization of rye ovarian tissue. Mol. Plant-Microbe Interact. 15:303-312.

Mitin, N., Rossman, K. L., and Der, C. J. 2005. Signaling interplay in Ras superfamily function. Curr. Biol. 16:R563-R574.

Müller, P., Katzenberger, J. D., Loubradou, G., and Kahmann, R. 2003. Guanyl nucleotide exchange factor Sq12 and Ras2 regulate filamentous growth in Ustilago maydis. Eukaryot. Cell 2:609-617.

Nishimura, M., Park, G., and Xu, J. R. 2003. The G-beta subunit MGB1 is involved in regulating multiple steps of infection-related morphogenesis in Magnaporthe grisea. Mol. Microbiol. 50:231-243.

Papadaki, P., Pizon, V., Onken, B., and Chang, E. C. 2002. Two Ras pathways in fission yeast are differentially regulated by two Ras guanine nucleotide exchange factors. Mol. Cell. Biol. 22:4598-4606.

Park, G., Xue, C., Zhao, X., Kim, Y., Orbach, M., and Xu, J. R. 2006. Multiple upstream signals converge on the adaptor protein Mst50 in Magnaporthe grisea. Plant Cell 18:2822-2835.

Parry, D. W., Jenkinson, P., and McLeod, L. 1995. Fusarium ear blight (scab) in small grain cereals - a review. Plant Pathol. 44:207-238.

Parton, R. G., and Hancock, J. F. 2004. Lipid rafts and plasma membrane microorganization: Insights from Ras. Trends Cell Biol. 14:141-147.

Phillips, A. J., Crowe, J. D., and Ramsdale, M. 2006. Ras pathway signaling accelerates programmed cell death in the pathogenic fungus Candida albicans. Proc. Natl. Acad. Sci. U.S.A. 103:726-731.

Pritsch, C., Muehlbauer, G. J., Bushnell, W. R., Somers, D. A., and Vance, C. P. 2000. Fungal development and induction of defense response genes during early infection of wheat spikes by Fusarium graminearum. Mol. Plant-Microbe Interact. 13:159-169.

Proctor, R. H., Hohn, T. M., and McCormick, S. P. 1995. Reduced virulence of Gibberella zeae caused by disruption of a trichothecene toxin biosynthetic gene. Mol. Plant-Microbe Interact. 8:593-601.

Ramezani-Rad, M. 2003. The role of adaptor protein Ste50-dependent regulation of the MAPKKK Ste11 in multiple signaling pathways of yeast. Curr. Genet. 43:161-170.

Ruiz-Roldan, M. C., Maier, F. J., and Schafer, W. 2001. PTK1, a mitogenactivated-protein kinase gene, is required for conidiation, appressorium formation, and pathogenicity of Pyrenophora teres on barley. Mol. Plant-Microbe Interact. 14:116-125.

Sambrook, J., and Russell, D. W. 2001. Molecular Cloning: A Laboratory Manual, third ed. Cold Spring Harbor Laboratory Press, Cold Spring Harbor, NY, U.S.A

Schubert, D., Raudaskoski, M., Knabe, N., and Kothe, E. 2006. Ras GTPase-activating protein Gap1 of the homobasidiomycete Schizophyllum commune regulates hyphal growth orientation and sexual development. Eukaryot. Cell 5:683-695.

Seong, K., Hou, Z. M., Tracy, M., Kistler, H. C., and Xu, J. R. 2005. Random insertional mutagenesis identifies genes associated with virulence in the wheat scab fungus Fusarium graminearum. Phytopathology 95:744-750.

Siafakas, A. R., Wright, L. C., Sorrell, T. C., and Djordjevic, J. T. 2006. Lipid rafts in Cryptococcus neformans concentrate the virulence determinants phospholipase $\mathrm{B} 1$ and $\mathrm{Cu} / \mathrm{Zn}$ superoxide dismutase. Eukaryot. Cell 5:488-498.

Solomon, P. S., Waters, O. D. C., Simmonds, J., Cooper, R. M., and Oliver, R. P. 2005. The Mak2 MAP kinase signal transduction pathway is required for pathogenicity in Stagonospora nodorum. Curr. Genet. 48:6068.

Sutton, J. C. 1982. Epidemiology of wheat head blight and maize ear rot caused by Fusarium graminearum. Can. J. Plant Pathol. 4:195-209.

Tanaka, K., Nakafuku, M., Satoh, T., Marshall, M. S., Gibbs, J. B. Matsumoto, K., Kaziro, Y., and Toh-e, A. 1990. S. cerevisiae genes IRA 1 and IRA2 encode proteins that may be functionally equivalent to mammalian ras GTPase activating protein. Cell 60:803-807.

Toda, T., Uno, I., Ishikawa, T., Powers, S., Kataoka, T., Broek, D., Cameron, S., Broach, J., Matsumoto, K., and Wigler, M. 1985. In yeast, Ras proteins are controlling elements of adenylate-cyclase. Cell 40:27-36.

Truckses, D. M., Bloomekatz, J. E., and Thorner, J. 2006. The RA domain of Ste 50 adaptor protein is required for delivery of Ste11 to the plasma membrane in the filamentous growth signaling pathway of the yeast Saccharomyces cerevisiae. Mol. Cell Biol. 26:912-928.

Ulrich, G., Seong, K. Y., Boddu, J., Cho, S. H., Trail, F., Xu, J. R., Adam, G., Mewes, H. W., Muehlbauer, G. J., and Kistler, H. C. 2006. Development of a Fusarium graminearum Affymetrix GeneChip for profiling fungal gene expression in vitro and in planta. Fungal Genet. Biol. 43:316-325.

Urban, M., Mott, E., Farley, T., and Hammond-Kosack, K. 2003. The Fusarium graminearum MAP1 gene is essential for pathogenicity and development of perithecia. Mol. Plant Pathol. 4:347-359.

van Egmond, H. P. 2002. Worldwide regulations for mycotoxins. Adv. Exp. Med. Biol. 504:257-269. 
Voigt, C. A., Schafer, W., and Salomon, S. 2005. A secreted lipase of Fusarium graminearum is a virulence factor required for infection of cereals. Plant J. 42:364-375.

Wanjiru, W. M., Kang, Z. S., and Buchenauer, H. 2002. Importance of cell wall degrading enzymes produced by Fusarium graminearum during infection of wheat heads. Eur. J. Plant Pathol. 108:803-810.

Waugh, M. S., Nichols, C. B., DeCesare, C. M., Cox, G. M., Heitman, J., and Alspaugh, J. A. 2002. Ras 1 and Ras2 contribute shared and unique roles in physiology and virulence of Cryptococcus neoformans. Microbiology 148:191-201.

Waugh, M. S., Vallim, M. A., Heitman, J., and Alspaugh, J. A. 2003. Ras1 controls pheromone expression and response during mating in Cryptococcus neoformans. Fungal Genet. Biol. 38:110-121.

Weeks, G., and Spiegelman, G. B. 2003. Roles played by Ras subfamily proteins in the cell and developmental biology of microorganisms. Cell. Signal. 15:901-909.

Wright, L. P., and Phillips, M. R. 2006. CAAX modification and targeting of Ras. J. Lipid Res. 47:883-891.
$\mathrm{Xu}$, J. R. 2000. MAP kinases in fungal pathogens. Fungal Genet. Biol. 31:137-152.

$\mathrm{Xu}$, J. R., and Hamer, J. E. 1996. MAP kinase and cAMP signaling regulate infection structure formation and pathogenic growth in the rice blast fungus Magnaporthe grisea. Genes Dev. 10:2696-2706.

Zhao, X. H., Xue, C., Kim, Y., and Xu, J. R. 2004. A ligation-PCR approach for generating gene replacement constructs in Magnaporthe grisea. Fungal Genet. Newsl. 51:17-18.

Zhao, X. H., Kim, Y., Park, G., and Xu, J. R. 2005. A mitogen-activated protein kinase cascade regulating infection-related morphogenesis in Magnaporthe grisea. Plant Cell 17:1317-1329.

\section{AUTHOR-RECOMMENDED INTERNET RESOURCE}

Broad Institute Fusarium graminearum database: www.broad.mit.edu/annotation/genome/fusarium_graminearum/Home. html 